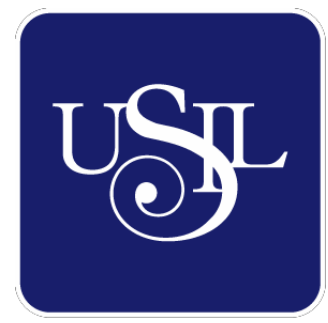

UNIVERSIDAD

SAN IGNACIO

DE LOYOLA

FACULTAD DE CIENCIAS EMPRESARIALES

Carrera de Gestión Ambiental Empresarial

\title{
EVALUACIÓN DE LOS DETERMINANTES DE PÉRDIDA DE BOSQUES, CRECIMIENTO URBANO, DEMANDA ELÉCTRICA Y PRODUCCIÓN DE ENERGÍA ELÉCTRICA EN EL PERÚ, PERIODO 2000-2016
}

Trabajo de investigación para obtener el Grado Académico de Bachiller en Gestión Ambiental Empresarial

JORGE ALONSO GARCÍA DE LA FUENTE

Asesor:

Carlos Enrique González Taranco

Lima - Perú

2018 


\section{Resumen}

El estudio analizó la relación que existe entre las variables dependientes: pérdida de bosques, crecimiento urbano, la demanda de energía eléctrica y la variable independiente producción de energía eléctrica en el Perú en el periodo 2000-2016 además, los factores que podrían influir en dicha valoración. Dentro de la metodología utilizada se consideraron 3 comparativas en función a la variable independiente; el consumo de energía eléctrica, población urbana y deforestación, estos en función a la producción de energía eléctrica. Estas variables fueron obtenidas del Instituto Nacional de Estadística e Informática (INEI), Ministerio del Ambiente (MINAM), Banco Central de Reserva del Perú (BCRP) y Organismo Supervisor de la Inversión en Energía y Minería (OSINERGMIN). Para la recolección de información se utilizó la base de datos históricos extraídos de estas entidades ya mencionadas con él método de Mínimos Cuadrados Ordinarios (MCO). Los resultados muestran que existe una correlación positiva por cada unidad de producción eléctrica la demanda eléctrica aumenta en 1.014341734, la perdida de bosques en 0.0274749350683 y la población urbana en - 0.0246743513831 . Se discute acerca de la relación entre las variables y el motivo de esta relación.

\section{Palabras claves:}

Deforestación, Crecimiento, Demanda, Consumo.

\section{Summary}

The study analyzed the relationship that exists between the dependent variables; forest loss, urban growth, the demand for electric power and the dependent variable electric power production in Peru in the period 2000-2016, in addition, the factors that could influence said valuation. Within the methodology used, 3 comparatives were considered according to the independent one; the consumption of electrical energy, urban population and deforestation, these depending on the production of electrical energy. These variables were obtained from the National Institute of Statistics and Informatics (INEI), the Ministry of the Environment (MINAM), the Central Reserve Bank of Peru (BCRP) and the Supervisory Body for Investment in Energy and Mining (OSINERGMIN). For the collection of information, the historical database extracted from these aforementioned entities was used with the Ordinary Least Squares (OLS) method. The results show that there is a positive correlation for each unit of electricity production, the electrical demand increases by 1.014341734 , the loss of forests by 0.0274749350683 and the urban population by -0.0246743513831 . The relationship between the variables and the reason for this relationship is discussed.

\section{Keywords:}

Deforestation, Growth, Demand, Consumption. 


\section{Introducción}

El planeta siempre ha tenido un constante crecimiento de la población humana, por lo mismo, nuestras actividades básicas y el consumo han ido en crecimiento contante e impactando de manera positiva como negativa en el medio ambiente, los ecosistemas, flora y fauna. Este impacto perjudica a la sociedad ya que depende de la provisión de recursos y servicios de los ecosistemas como: secuestro de dióxido de carbono, regulador climático, producción de oxígeno, entre otros.

Como lo menciona la CEPAL desde 1950 hacia el 2050 la población mundial rural va a estar en decrecimiento mientras que la población urbana mundial ha tenido un crecimiento exponencial, esto significa que, la población rural ha migrado hacia las ciudades. La proyección de la CEPAL supone que para el año 2050 menos del $20 \%$ de la población será rural.

Gráfica. 1

\section{POBLACIÓN RURAL SEGŨN GRANDES REGIONES DEL MUNDO, 1950-2050}

(En porcentajes)

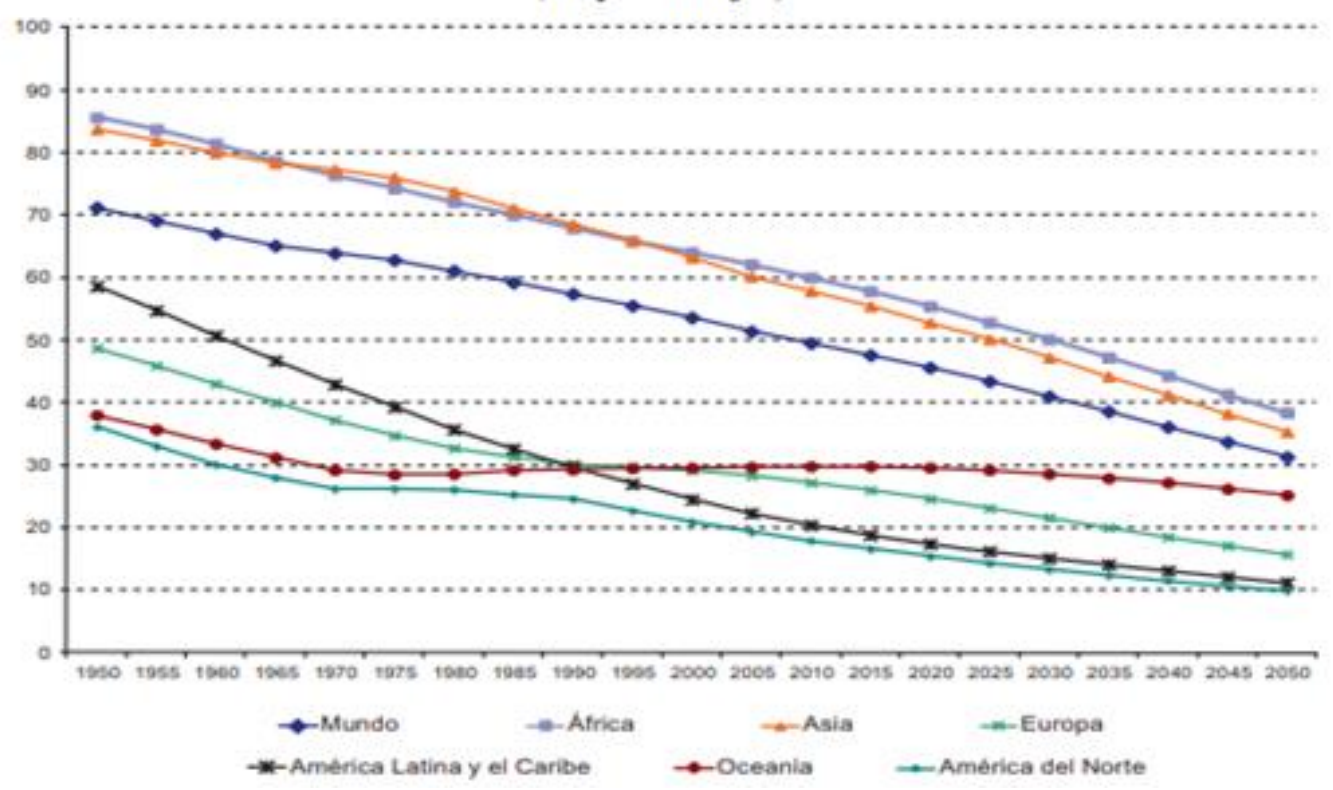

(Fuente: Naciones Unidas, World Urbanization Prospects: The 2010 Revision)

Esto supondría que, en países como el Perú, se vea un gran aumento en la población urbana en ciudades como Lima, Arequipa, Trujillo y Cuzco, que son las más grandes del país.

Las ciudades van a un ritmo de crecimiento poblacional rápido y la demanda eléctrica va a estar relacionada con el aumento de la población urbana de un país, así como su crecimiento, ya que una urbe consume más energía que una zona rural, porque hay un mayor desarrollo de tecnosfera la cual requiere de energía para su funcionamiento, a 
ello se suma, el incremento del número de personas con mayor poder adquisitivo que demanda un mayor consumo de energía eléctrica.

Respecto a la electricidad y sus fuentes en el Perú, se identifica que $41 \%$ de la electricidad generada proviene de energía hídrica y el $53.1 \%$ de hidrocarburos (principalmente gas) como se muestra en la Gráfica 2. Esta relación varía durante el año con el nivel del agua en las represas: por ejemplo, de diciembre a junio la generación hidroeléctrica es mayor debido a al aumento de lluvias y al aumento del caudal.

Gráfica. 2

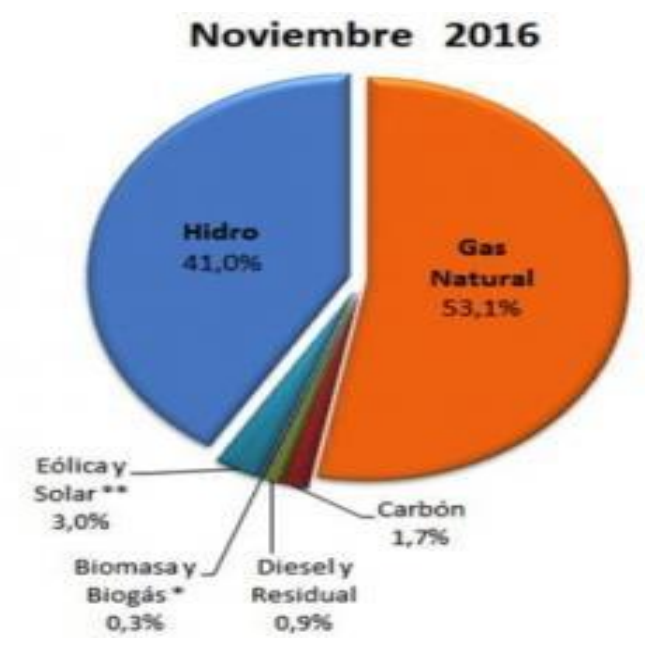

Fuente: (Avance Estadístico del Subsector Eléctrico, MINEM).

Actualmente, el país posee una capacidad alta de generación eléctrica la cual se encuentra por encima de la demanda eléctrica. Según el informe de la MRFO 2016 la producción máxima del Sistema Eléctrico Interconectado Nacional del Perú (SEIN) es de 11.22GW mientras que la demanda es de 6.67GW (COES, Informe MRFO 2016).

Como menciona el Organismo Supervisor de la Inversión en Energía y Minería (Osinergmin) y la Revista Rumbo Minero, el Perú cuenta con la una tarifa eléctrica de un $32 \%$ por debajo del promedio en toda la región de Latinoamérica en el costo eléctrico y con una proyección de que estos precios serán más competitivos en el futuro. 
Gráfica. 3

Tarifas de energía eléctrica para la industria en 2015

(centavos de dólar por Kwh)

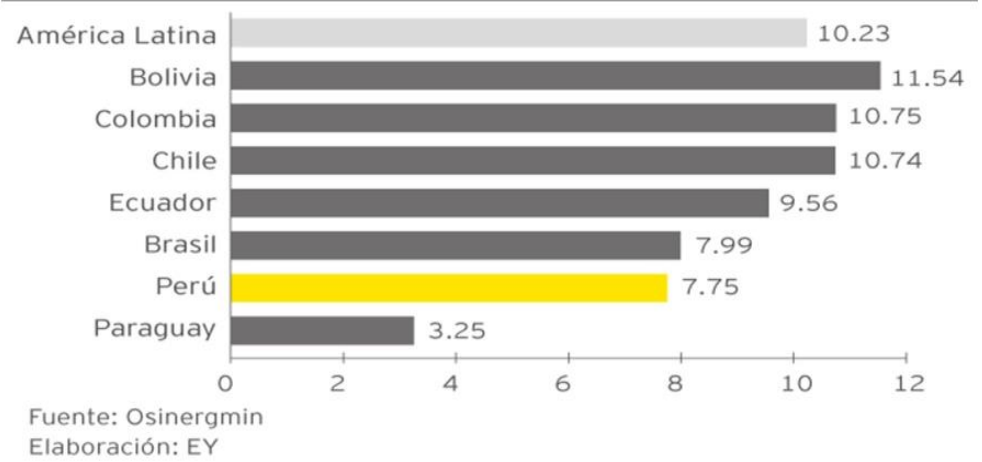

Como se muestra en la Gráfica 3, Perú siempre ha tenido un precio competitivo de tarifa eléctrica, esto supone que el consumo de energía eléctrica en nuestro país tanto en industrias como en los hogares sea mayor ya que las personas no toman conciencia de su importancia para la sociedad y solo la malgastan por su fácil acceso al tener un precio bajo. En cambio, en otros países de la región como Chile, la tarifa eléctrica es más elevada por lo cual buscan ahorrar este recurso para no generar gastos innecesarios.

Por otro lado, la población urbana en el Perú está en constante crecimiento, esto se debe por las grandes migraciones de las zonas rurales a zonas urbanas como es el caso de Lima; como se puede ver en la Gráfica 4 el $98 \%$ de su población vive en zonas urbanas.

Gráfica. 4

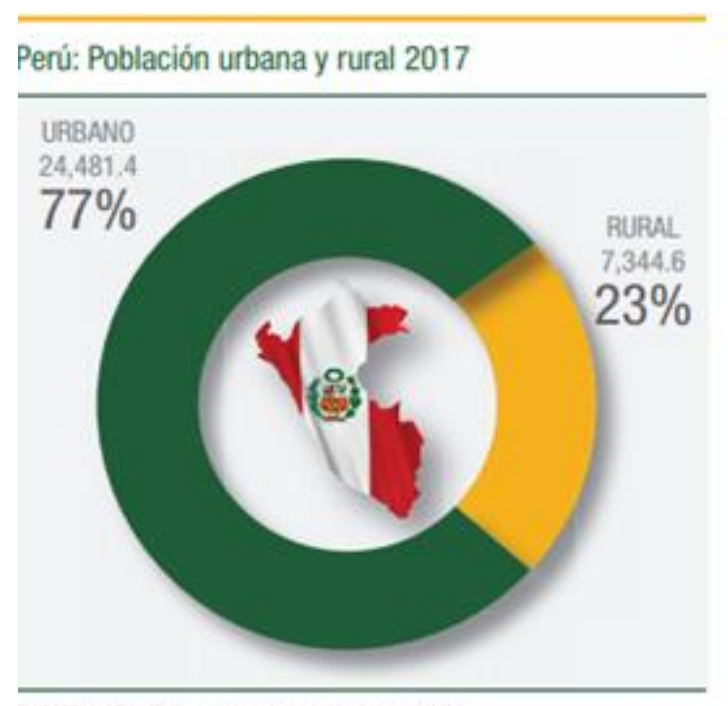

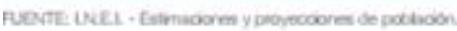

Fuente: INEI, 2017.

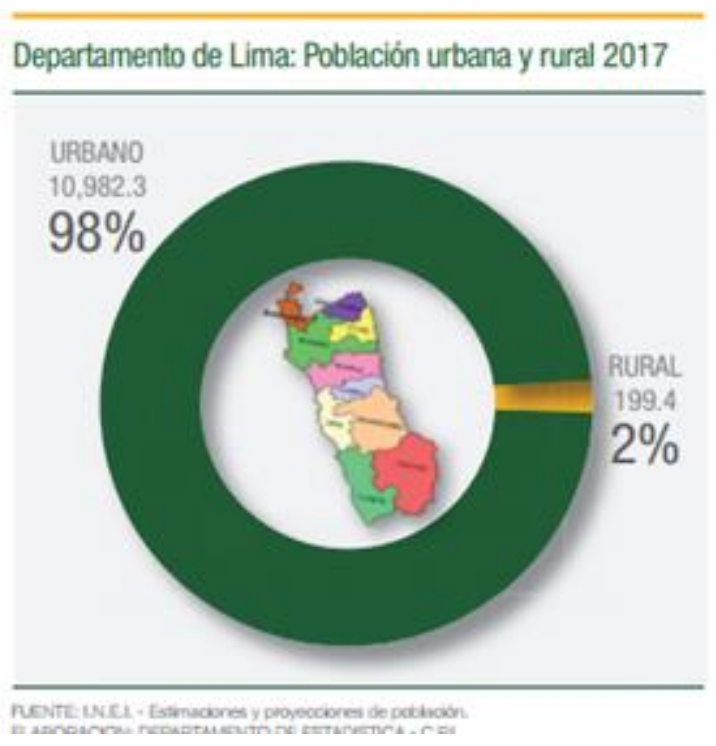


En la Gráfica 4 se observa la población urbana y rural al 2017 y como la población rural en nuestro país es cada vez menos, un ejemplo es el departamento de Lima donde solo el $2 \%$ de la población vive en zonas rurales y a nivel nacional el $23 \%$ vive en zonas rurales esto ocurre porque las ciudades están en constante crecimiento debido a la migración interna.

Todo crecimiento poblacional en ciudades tiende a extender su territorio, también demanda mayor cantidad de recursos del ecosistema y una mayor cantidad de energía eléctrica

La deforestación en el Perú sigue siendo uno de los principales problemas por el elevado número de hectáreas de bosques perdidos y ecosistemas afectados.

La deforestación alcanzó su nivel más alto de los últimos años el 2014 (177 566 hectáreas deforestadas). Esta información fue dada a conocer por el Servicio Nacional Forestal y de Fauna Silvestre Ministerio de Agricultura (MINAGRI) y el Programa Nacional de Conservación de Bosques para la Mitigación del Cambio Climático Ministerio del Ambiente (MINAM), quienes presentaron oficialmente los datos de pérdida de cobertura de los bosques húmedos amazónicos en el 2016.

El problema que se va a abordar en el estudio es:

¿Existe relación entre la producción de energía eléctrica con respecto al consumo de energía eléctrica, población urbana y la perdida de bosques en el Perú, durante el periodo 2000 al $2016 ?$

Al existir un aumento en la población urbana en Lima y otras ciudades del Perú, estas ciudades van a expandirse y afectar zonas como los bosques por el aumento de la demanda de recursos. La afectación de los bosques se da también con el aumento en el consumo de la energía eléctrica ya que esta va provocar que se creen más hidroeléctricas en el país lo que significará impactar en diferentes tipos de ecosistemas como los bosques.

Se busca analizar cómo la producción eléctrica ya sea positiva o negativa va afectar al crecimiento urbano, la demanda eléctrica y perdida de bosques en el Perú.

El crecimiento poblacional se ve sustentado por proyecciones de crecimiento del Instituto Nacional de Estadística e Informática (INEI) en un trabajo de proyecciones de 1990 a 2025 y su metodología. Los datos de venta y producción de energía eléctrica son extraídos del Banco Central de Reserva del Perú (BCRP). 
Asimismo, las variables a evaluar en esta investigación corresponden al periodo 20002016 en un contexto de crecimiento de las zonas urbanas, demanda eléctrica, así como la creación de distintas instituciones gubernamentales que buscan proteger los recursos naturales como los bosques y racionar el uso de la energía eléctrica.

\section{Método}

\subsection{Tipo y diseño de investigación}

\subsubsection{Tipo de investigación}

Este proyecto se trabajó con el método de Mínimos Cuadrados Ordinarios (MCO), basándose en la recolección de datos confiables. La investigación es de tipo cuantitativo eso quiere decir que se utilizan solamente datos numéricos. El objetivo principal de la investigación es analizar si existe alguna relación entre la producción de energía eléctrica con respecto a venta de energía eléctrica, población urbana y la perdida de bosques. Las variables que se van a utilizar son anuales.

\subsubsection{Diseño de investigación}

El tipo de investigación es no experimental con un diseño correlacional.

Se plantea el siguiente modelo de regresión lineal simple:

$$
p e e=v e e+p u+p b+U \text {. }
$$

Donde:

pee: Producción de energía eléctrica.

vee: Venta (consumo) de energía eléctrica.

pu: Población urbana.

pb: Pérdida de bosques.

U: Error estocástico.

También, se plantea el siguiente modelo de regresión lineal simple de tipo log - log debido a que los valores son grandes y es necesario reducirlas.

$$
I p e e=I v e e+I p u+I p b+U .
$$

Donde:

pee: logaritmo de la producción de energía eléctrica.

vee: logaritmo de la venta de energía eléctrica.

pu: logaritmo de la población urbana. 
pb: log de la pérdida de bosques.

U: error estocástico.

\subsection{Participantes}

Venta de energía eléctrica: vee

Cantidad de Giga watts vendidos de energía eléctrica en el Perú, periodo 2000- 2016.

\begin{tabular}{|c|c|c|}
\hline Unidad & Periodo de ocurrencia & Técnica \\
\hline GigaWatt & anual & Ponderación \\
\hline
\end{tabular}

Fuente: Banco Central de Reserva del Perú (BCRP).

Producción de energía eléctrica: pee

Cantidad de Giga watts producidos de energía eléctrica en el Perú, periodo 2000- 2016.

\begin{tabular}{|c|c|c|}
\hline Unidad & Periodo de ocurrencia & Técnica \\
\hline GigaWatt & anual & Ponderación \\
\hline
\end{tabular}

Fuente: Banco Central de Reserva del Perú (BCRP).

Población urbana en el Perú: pu

Población urbana a nivel nacional para los años comprendidos del 2000 al 2016.

\begin{tabular}{|c|c|c|}
\hline Unidad & Periodo de ocurrencia & Técnica \\
\hline Miles de habitantes. & anual & Ponderación \\
\hline
\end{tabular}

Fuente: Instituto Nacional de Estadística e Informática (INEI).

\section{Perdida de bosques en el Perú: pb}

La cantidad de hectáreas perdidas por la deforestación en el Perú en el periodo 20002016.

\begin{tabular}{|c|c|c|}
\hline Unidad & Periodo de ocurrencia & Técnica \\
\hline Hectáreas & anual & Ponderación \\
\hline
\end{tabular}

Fuente: Ministerio de Agricultura (MINAGRI) y Ministerio del Ambiente (MINAM). 


\subsection{Instrumentos}

Los instrumentos que se utilizo fue el de recolección de datos cuantitativos.

Este proceso en cuanto a la relación de la población urbana y la demanda eléctrica ya tuvo lugar en investigaciones pasadas tanto del Banco Central de Reserva del Perú, como en las proyecciones de OSINERG e INEI, por lo que estos datos ya fueron extraídos con total confianza de los modelos propuestos por estas instituciones. La variable deforestación fue obtenida de datos de investigaciones del MINAGRI y MINAM en comparativas de deforestación en la línea de tiempo.

\subsection{Procedimiento}

Procedimientos de recolección de datos:

La forma en que desarrolló el proceso de recolección de datos consistió en una extracción de data de la base de datos estadística del Banco Central de Reserva del Perú (BCRP), Instituto Nacional de Estadística e Informática (INEI) y de la base de datos de deforestación de los últimos años del Ministerio de Agricultura (MINAGRI) Y del Ministerio del Ambiente (MINAM) del 2000 - 2016.

Los datos de venta y producción de energía eléctrica en el Perú fueron conseguidos en tasas mensuales por lo que fue necesario la conversión a anuales.

Respecto a, los datos de deforestación se obtuvieron en datos anuales e incompletos por lo cual fue necesario complementar los datos y combinar la data del Ministerio del Ambiente (MINAM), con la del Ministerio de Agricultura (MINAGRI) y de esa forma se obtuvo una base de los años del 2000 - 2016 confiable.

Con respecto a la data de población urbana esta se obtuvo del Instituto Nacional de Estadística e informática (INEI). Esta recolección fue de las proyecciones anuales del INEI, en la que se mostraba por departamento el porcentaje de población urbana y rural por lo que fue necesario hacer la sumaria total para saber la data nacional de población urbana.

\subsection{Análisis de datos}

El proyecto sigue un análisis cuantitativo y tendrá un enfoque estadístico - econométrico para el tratamiento de sus datos. El método de estimación econométrica elegido para este trabajo, dada la naturaleza de los datos recolectados, será el de un modelo de 
Mínimos Cuadrados Ordinarios (MCO), la cual relaciona las variables tomadas para el desarrollo de la investigación.

\section{Resultados}

\section{Presentación de Resultados:}

Estimación de Parámetros y Prueba estadísticos

Ecuación estimada:

\section{LPEE $=1.014341734^{\star}$ LVEE $+0.0274749350683^{\star}$ LPB $-0.0246743513831^{\star}$ LPU}

\begin{tabular}{|c|c|c|c|c|}
\hline \multicolumn{5}{|c|}{$\begin{array}{l}\text { Dependent Variable: LPEE } \\
\text { Method: Least Squares } \\
\text { Date: } 11 / 27 / 17 \text { Time: } 17: 24 \\
\text { Sample: } 20002016 \\
\text { Included observations: } 17\end{array}$} \\
\hline Variable & Coefficient & Std. Error & t-Statistic & Prob. \\
\hline $\begin{array}{l}\text { LVEE } \\
\text { LPB } \\
\text { LPU }\end{array}$ & $\begin{array}{r}1.014342 \\
0.027475 \\
-0.024674\end{array}$ & $\begin{array}{l}0.049697 \\
0.046900 \\
0.039661\end{array}$ & $\begin{array}{r}20.41055 \\
0.585820 \\
-0.622126\end{array}$ & $\begin{array}{l}0.0000 \\
0.5673 \\
0.5439\end{array}$ \\
\hline $\begin{array}{l}\text { R-squared } \\
\text { Adjusted R-squared }\end{array}$ & $\begin{array}{l}0.992689 \\
0.991644\end{array}$ & \multicolumn{2}{|c|}{$\begin{array}{l}\text { Mean dependent var } \\
\text { S.D. dependent var }\end{array}$} & $\begin{array}{l}10.37780 \\
0.334158\end{array}$ \\
\hline S.E. of regression & 0.030545 & \multicolumn{2}{|c|}{ Akaike info criterion } & 3.980443 \\
\hline Sum squared resid & 0.013062 & \multicolumn{2}{|c|}{ Schwarz criterion } & 3.833406 \\
\hline $\begin{array}{l}\text { Log likelihood } \\
\text { Durbin-Watson stat }\end{array}$ & $\begin{array}{l}36.83377 \\
0.335261\end{array}$ & \multicolumn{2}{|c|}{ Hannan-Quinn criter. } & 3.965827 \\
\hline
\end{tabular}

Elaboración propia.

Formula:

LPEE $=1.014341734^{*}$ LVEE $+0.0274749350683^{*}$ LPB $-0.0246743513831^{*}$ LPU

Se concluye que la variación entre producción de energía eléctrica (LPPE) y venta de energía eléctrica (LVEE) tiene una relación positiva ya que por cada unidad de GWh de energía producida se demanda 1.014341734 veces lo que se produce.

Se concluye que la variación entre producción de energía eléctrica (LPPE) y población urbana (LPU) tiene una relación negativa ya que por cada unidad de GWh de energía 
producida existe un decrecimiento poblacional de -0.0246743513831 miles de personas.

Se concluye que la variación entre producción de energía eléctrica (LPPE) y pérdida de bosques (LPB) tiene una relación positiva ya que por cada unidad de GWh de energía producida existe un decrecimiento de 0.0274749350683 hectáreas.

\section{Discusión}

La relación entre la producción eléctrica y perdida de bosques es positiva esto es posible porque se tiene casi el 50\% de energía eléctrica producida por hidroeléctricas en el Perú lo que supone una disminución en el impacto de la deforestación ya que una vez construida la hidroeléctrica ya no generan impactos de deforestación, porqué tiene un impacto primario en las actividades de construcción.

En el 2008 se creó el Ministerio del Ambiente, también, diversas instituciones públicas relacionadas al tema ambiental como el Organismo de Evaluación y Fiscalización Ambiental (OEFA) los cuales contribuyeron a la gobernanza ambiental desarrollando acciones para el cuidado y conservación del ambiente y los recursos naturales. Pese a esto, la relación de perdida de bosques sigue siendo positiva por lo cual actualmente se siguen perdiendo muchas hectáreas de bosque en nuestro país, como en los departamentos de Madre de Dios y San Martin que presentan la mayor tasa de deforestación.

Respecto a la relación negativa entre la producción eléctrica y población urbana esta puede ser justificada debido a que en muchos de los departamentos se están teniendo alto crecimientos poblacionales en los últimos años a pesar de no ser ciudades consolidadas como Lima, eso quiere decir que, actualmente existe un alto porcentaje de crecimiento poblacional tanto en zonas urbanas como rurales. La relación negativa entre la producción eléctrica y población urbana tiende a convertirse en una relación positiva con lo cual en un futuro el crecimiento urbano se relacionará positivamente con la producción eléctrica. Esta tendencia se ve justificada por la constante migración de las personas a las ciudades y la creación de nuevos proyectos de urbanización en zonas periurbanas que se crean contantemente tanto por empresas privadas como Los Portales y el apoyo del Estado Peruano atreves de Mi Vivienda.

Se recomienda buscar una mayor cantidad de datos para ampliar el horizonte de años, de esa forma poder tener un mejor tratamiento de las variables y tener resultados con mayor exactitud para el análisis. 
Finalmente, se recomienda utilizar otras variables de medición ambiental como la cantidad de $\mathrm{CO}^{2}$ producido, la perdida de biomasa, contaminación de lagos y ríos para conocer de qué manera la producción eléctrica impacta en otros aspectos ambientales. 


\section{Referencias}

Ahmed K. y Shahbaz M. (2015), The linkages between deforestation, energy and growth for environmental degradation in Pakistan, Recuperado de http://www.sciencedirect.com/science/article/pii/S1470160X14004725

Beltrán A. y Morera M. y López F. y Villela R. (2017). Prosperidad de las energías eólicas y solar fotovoltaicas en la producción de energía eléctrica, Recuperado de http://www.scielo.org.mx/pdf/cuat/v11n2/2007-7858-cuat-11-02-00105.pdf

Chervier C. y Costedoat S. (2017), Heterogeneous Impact of a Collective Payment for Environmental Services Scheme on Reducing Deforestation in Cambodia, Recuperado

de http://www.sciencedirect.com/science/article/pii/S0305750X15311864

Cardoso J. y Prasad S. y Felizalo J. (2017). The impact of deforestation, urbanization, public investments, and agriculture on human welfare in the Brazilian Amazonia, Recuperado de http://www.sciencedirect.com/science/article/pii/S0264837716307256

Cayetano E. y García M. (2012). La energía eólica en la producción de electricidad en España, Recuperado de http://www.scielo.cl/pdf/rgeong/n51/art07.pdf

Fang Z. y Chen Y. (2017). Industrial electricity consumption, human capital investment and economic growth in Chinese cities, Recuperado de http://www.sciencedirect.com/science/article/pii/S0264999317304340

García M. y Moreno B. (2015). La liberalización en la industria eléctrica española, Recuperado de http://www.scielo.org.mx/pdf/gpp/v25n2/1405-1079-gpp-25-0200551.pdf

Ghazoul J. (2016), Deforestation and Land Clearing, recuperado de http://www.sciencedirect.com/science/article/pii/B9780123847195002811

Li C. y Yan Song Y Kaza N. (2017). Urban form and household electricity consumption: A multilevel study, Recuperado de http://www.sciencedirect.com/science/article/pii/S0378778817321436

Machicao J. y Olazabal J. MATRIZ ENERGÉTICA EN EL PERÚ Y ENERGÍAS RENOVABLES, recuperado de http://library.fes.de/pdffiles/bueros/peru/10182.pdf 
Mark T. y Stuart E. (2015), Titling community land to prevent deforestation: An evaluation of a best-case program in Morona-Santiago, Ecuador, recuperado de http://www.sciencedirect.com/science/article/pii/S0959378015000503

Rodríguez P. y Sarmiento L. (2016). Análisis de costos de la generación de energía eléctrica mediante fuentes renovables en el sistema eléctrico colombiano, recuperado

de http://www.scielo.org.co/scielo.php?script=sci arttext\&pid=S0122$34612016000200008 \& \operatorname{lng}=p t \& \operatorname{lng}=e s$

Shaaban M. y Scheffran J. (2017), Selection of sustainable development indicators for the assessment of electricity production in Egypt, Recuperado de http://www.sciencedirect.com/science/article/pii/S221313881730365X

Tiruwork y T. Tewodros (2016), Household willingness to pay for green electricity in urban and peri-urban Tigray, northern Ethiopia: Determinants and welfare effects, Recuperado de http://www.sciencedirect.com/science/article/pii/S0301421516305675

Torres A. y Matínez E. (2015). Evaluación de confiabilidad tecnológica del parque aerogenerador de Gibara, recuperado de http://scielo.sld.cu/pdf/rie/v37n1/rie04116.pdf

Vargas E. (2017). HACIA UN SISTEMA DE PROPIEDAD PRIVADA SOBRE EL VIENTO EN CHILE, Recuperado de http://www.scielo.cl/pdf/rchilder/v44n1/art02.pdf

Zaim O. y Taskin F. (2016). Environmental efficiency in carbon dioxide emissions in the OECD: $\quad A$ non-parametric approach, Recuperado de http://www.sciencedirect.com/science/article/pii/S0301479799903122

Ministerio del Ambiente (2017) Sistema Nacional de Gestión Ambiental, Recuperado de http://www.minam.gob.pe/gestion-ambiental/

By Mexican and Spanish Ministries of Environment (2010) OECD Environmental Outlook to 2030 http://www.oecd.org/env/indicators-modelling-outlooks/40224072.pdf

Zhang C. Y Zhou K. y Yang S. y Shao Z. (2017) On electricity consumption and economic growth in China, Recuperado de http://www.sciencedirect.com/science/article/pii/S1364032117304045 